\title{
An improved version of the Hughes model for pedestrian flow
}

\author{
Jose A. Carrillo \\ Department of Mathematics, Imperial College London, \\ London $S W 72 A Z, U K$ \\ carrillo@imperial.ac.uk \\ Stephan Martin \\ Department of Mathematics RWTH Aachen University, \\ 52074 Aachen, Germany \\ Department of Mathematics, Imperial College London, \\ London SW7 2AZ, UK \\ martin@mathcces.rwth-aachen.de \\ stephan.martin@imperial.ac.uk \\ Marie-Therese Wolfram \\ Radon Institute for Computational and Applied Mathematics, \\ Austrian Academy of Sciences, Altenberger Strasse 69, \\ 4040 Linz, Austria \\ mt.wolfram@ricam.oeaw.ac.at
}

Received 28 January 2015

Revised 9 September 2015

Accepted 25 September 2015

Published 19 January 2016

Communicated by N. Bellomo

Roger Hughes proposed a macroscopic model for pedestrian dynamics, in which individuals seek to minimize their travel time but try to avoid regions of high density. One of the basic assumptions is that the overall density of the crowd is known to every agent. In this paper we present a modification of the Hughes model to include local effects, namely limited vision, and a conviction towards decision making. The modified velocity field enables smooth turning and temporary waiting behavior. We discuss the modeling in the micro- and macroscopic setting as well as the efficient numerical simulation of either

This is an open access article published by World Scientific Publishing and distributed under the terms of the Creative Commons Attribution (CC BY) 4.0 License, which permits use, distribution and reproduction in any medium, provided the original author(s) and source are credited. 
description. Finally we illustrate the model with various numerical experiments and evaluate the behavior with respect to the evacuation time and the overall performance.

Keywords: Hughes model; crowd dynamics; local vision; eikonal equations; selforganization.

AMS Subject Classification: 35Q91, 35Q70, 35F21, 91C99

\section{Introduction}

The mathematical modeling and simulation of pedestrian dynamics, such as large human crowds in public space or buildings, has become a topic of high practical relevance. The complex behavior of these large crowds poses significant challenges on the modeling, analytic and simulation level. These aspects initiated a lot of research in the mathematical community within the last years, which we briefly outline below. Mathematical modeling approaches for pedestrian dynamics can be roughly grouped into the following categories:

(1) Microscopic models such as the social force model ${ }^{25,24,38}$ or cellular automata approaches. $^{11}$

(2) Fluid dynamic approaches ${ }^{12,33,4}$ and related macroscopic models, see for example the popular Hughes model. ${ }^{29,18,23,10,39}$

(3) Kinetic models ${ }^{6,17}$ which uses ideas from gas kinetics to models interactions between individuals via so-called collisions.

(4) In optimal control ${ }^{27}$ and mean-field game approaches ${ }^{31,19}$ pedestrians act as rational individuals, which adjust their velocity optimal to a specific cost.

(5) Multiscale models coupling between different scales to describe for example crowd leader dynamics. ${ }^{15,8}$

A detailed survey on crowd modeling can e.g. be found in Ref. 7. Several aspects are considered to be important in the mathematical modeling to capture the complex behavior in a correct way. For example, repulsive forces when getting too close to other individuals or obstacles play an important role in the dynamics. Another popular assumption is the fact that individuals act rationally and try to make the optimal decision based on their actual knowledge level. Partial knowledge of the overall pedestrian density or the domain is another important factor which should be taken into account in the modeling. While these nonlocal effects can be implemented quite intuitively on the microscopic level, their translation for macroscopic models is not straightforward. Most macroscopic nonlocal models are based on the continuity equation for the pedestrian density, where the nonlocal effects correspond to the deviation of the crowd from its preferred direction. ${ }^{12-14}$ This deviation is determined by the average density felt by the pedestrians and modeled via a convolution operator acting on the velocity. The development of numerical schemes for conservation laws with nonlocal effects gained substantial interest in the last years. This was, among other factors, also initiated by the development of nonlocal models in traffic flow. ${ }^{3,9}$ 
The original model of Hughes ${ }^{29}$ describes fast exit and evacuation scenarios, where a group of people wants to leave a domain $\Omega \subset \mathbb{R}^{2}$ with one or several exits/doors and/or obstacles as fast as possible. The driving force towards the exit is the gradient of a potential $\phi=\phi(x, t), x \in \Omega, t>0$. This potential corresponds to the expected travel time to maneuver through the present pedestrian density towards an exit. Hughes assumed that the global distribution of pedestrians is known to every individual, an assumption not generally satisfied in real world applications.

In this paper we present a generalization of the classical Hughes model, which includes local vision via partial knowledge of the pedestrian density. We discuss the proper modeling setup, the implementation of suitable numerical schemes as well as their computational complexity. Furthermore we compare how the reduced perception of each pedestrian affects the overall "performance" of the crowd in evacuation scenarios. Inevitably, one expects the crowd to behave less efficient as less information is available. Quantifying how localized vision influences performance and decision making is a very interesting question in terms of collective behavior. Surprisingly, it will turn out that evacuation times can even improve. The question we investigate is therefore complementary to mean-field game approaches, where pedestrians anticipate future crowds states and hence are more capable than in the original Hughes' model. ${ }^{31,19,10}$

This paper is structured as follows. We start with a review on the modeling and analytic results of the classical Hughes model for pedestrian flow and its microscopic interpretation in Sec. 2. In Sec. 3 we present the local version of the Hughes model on the micro- and macroscopic level. Section 4 presents the numerical strategies for the microscopic and macroscopic model. We compare the behavior and performance of the models in Sec. 5 and conclude with a discussion of the proposed model in Sec. 6 .

\section{Hughes' Model for Pedestrian Flow}

\subsection{Original formulation and analytic results}

Let us start by presenting the original modeling assumptions and the corresponding partial differential equation system of the Hughes model for pedestrian flow. Hughes considered an exit scenario, in which a crowd modeled by a macroscopic density $\rho=\rho(x, t)$ wants to leave a domain as fast as possible. The nonlinear PDE system for $\rho$ and the potential $\phi=\phi(x, t)$ on the domain $\Omega \subset \mathbb{R}^{2}$ read as:

$$
\begin{aligned}
\frac{\partial \rho}{\partial t}-\operatorname{div}\left(\rho f(\rho)^{2} \nabla \phi\right) & =0, \\
\|\nabla \phi\| & =\frac{1}{f(\rho)} .
\end{aligned}
$$

The first equation describes the evolution of $\rho$ in time, driven by the gradient of $\phi$ and weighted by a nonlinear mobility $f=f(\rho)$. This mobility includes saturation effects, i.e. degenerate behavior when approaching a given maximum density 
$\rho_{\max } \in \mathbb{R}^{+}$. Possible choices are $f(\rho)=\rho-\rho_{\max }$ or $f(\rho)=\left(\rho-\rho_{\max }\right)^{2}$ amongst others. The former is inherited from the Lighthill-Whitham-Richards model for one-dimensional traffic flow. ${ }^{32,35}$

The potential $\phi$ corresponds to the weighted shortest distance to an exit in the following sense: Solving the eikonal equation (2.1b) determines the optimal path $\nabla \phi$ minimizing the expected travel time throughout the crowd towards an exit. This cost is measured as the inverse of $f(\rho)$, hence the cost of walking through dense regions is high. Equation (2.1b) is also a stationary Hamilton-Jacobi-Bellman equation, and the optimal path property of $\nabla \phi$ can be rigorously derived. ${ }^{5,26}$ The fact that the potential $\phi$ solely determines the direction of the flow can be easily seen as $f^{2}(\rho) \nabla \phi=f(\rho) \nabla \phi /\|\nabla \phi\|$ using (2.1b).

Hughes model (2.1) is supplemented with different boundary conditions for the walls and the exits. We assume that the boundary is divided into two parts: either impenetrable walls $\partial \Omega_{\text {wall }} \subset \partial \Omega$ or exits/doors $\partial \Omega_{\text {exit }} \subset \partial \Omega$, with $\partial \Omega_{\text {wall }} \cap \partial \Omega_{\text {exit }}=$ $\emptyset$. Typical conditions for the density $\rho$ in (2.1) are zero flux boundary conditions at $\partial \Omega_{\text {wall }}$, which are either automatically satisfied as $\nabla \phi \cdot \mathbf{n}=0$ or artificially enforced. The flux at $\partial \Omega_{\text {exit }}$ has to be defined according to the arriving density and our choices are discussed in Sec. 3. The boundary conditions of (2.1b) are set as $\phi(x, t)=0$ for all $x \in \partial \Omega_{\text {exit }}$.

There has been a lot of recent mathematical research on the classical Hughes model. ${ }^{18,23,1,21,2}$ Up to the authors knowledge all analytic results are restricted to $1 \mathrm{D}$ only, which is caused by the low regularity of the potential $\phi$. This low regularity, i.e. $\phi \in C^{0,1}$, results in the formation of shocks and rarefaction waves in the conservation law. It is caused particularly by the existence of sonic points, which are hypersurfaces in space, where costs towards two or more exits coincide, and therefore $\nabla \phi$ does not exist and the orientational field is discontinuous. In spatial dimension one the system can be reduced to the conservation law with a discontinuous flux function. Global existence of entropy solutions in 1D has been obtained in Ref. 2 under suitable assumptions which guarantee a good analytical behavior of the sonic point. In this case it is possible to solve the corresponding Riemann problem, ${ }^{18}$ which also serves as a basis for different numerical schemes. ${ }^{23,9}$

\subsection{Microscopic interpretation}

Hughes motivated system (2.1) on the macroscopic level only. Recently Burger et $a l .{ }^{10}$ were able to give a microscopic interpretation of $(2.1)$, which will serve as a basis for our local particle model. Microscopic models based on Hughes' modeling assumptions are also used in the field of computer vision. ${ }^{38}$

Let us consider $N$ particles with position $X^{j}=X^{j}(t)$ and velocity $V^{j}=V^{j}(t)$, $j=1, \ldots, N$. Then the empirical density $\rho^{N}=\rho^{N}(t)$ is given by

$$
\rho^{N}(t)=\frac{1}{N} \sum_{j=1}^{N} \delta\left(x-X^{j}(t)\right) .
$$


Furthermore we introduce its smoothed approximation $\rho_{g}^{N}=\rho_{g}^{N}(t)$, given by

$$
\rho_{g}^{N}(x, t)=\left(\rho^{N} * g\right)(x, t)=\frac{1}{N} \sum_{j=1}^{N} g\left(x-X^{j}(t)\right),
$$

where the function $g=g(x)$ corresponds to a sufficiently smooth positive kernel. The walking cost is given by the sum of a weighted kinetic energy and the exit time, defined as $T_{\text {exit }}=\sup \{t>0 \mid x \in \Omega\}$. Then the problem reads as:

$$
\begin{aligned}
& C(X ; \rho(t))=\min _{(X, V)} \frac{1}{2} \int_{t}^{T+t} \frac{\|V(s)\|^{2}}{f^{2}\left(\rho_{g}^{N}(\xi(s ; t), t)\right)} d s+\frac{1}{2} T_{\text {exit }}(X, V), \\
& \text { subject to } \frac{d \xi}{d s}=V(s) \text { and } \xi(0)=X(t) .
\end{aligned}
$$

Hence the optimal trajectory is determined by "freezing" the empirical density $\rho^{N}=\rho^{N}(t)$, in other words it corresponds to extrapolating the empirical density $\rho^{N}$ in time when looking for the optimal trajectory.

Burger et $a l .{ }^{10}$ were able to show that Hughes' model can be formally derived from the optimality conditions of $(2.3)$ and letting $T \rightarrow 0$ (corresponding to the long-time behavior of the corresponding adjoint Hamilton-Jacobi equation).

We will use this microscopic interpretation to propose a numerical approximation by a particle method in Sec. 4 of Hughes-type models. In fact, (2.1a) is seen as a continuity equation with velocity field $v(x, t)=-f(\rho)^{2} \nabla \phi$ driven by $(2.1 \mathrm{~b})$, and thus particles in (2.2) are advected by the velocity field $v$, e.g.

$$
\frac{d X^{j}}{d t}=v\left(X^{j}(t), t\right), \quad j=1, \ldots, N
$$

\section{A Localized Smooth Hughes-Type Model for Pedestrian Flow}

The Hughes model (2.1) assumes that at any time $t>0$ the global distribution of all other individuals $\rho(x, t)$ is known to every pedestrian. Therefore she chooses her optimal walking direction $\nabla \phi$ in order to minimize its expected travel time/costs. Here, all walking costs are based on the current density, which means that pedestrians do not anticipate future dynamics of the crowd. Instead they are capable to react to changes in the global density ad hoc as the path optimization is repeated continuously in time. In a mean-field game-type model, the capabilities of pedestrians would increase, as the planning decision of all agents can be correctly predicted into the future. We follow an opposite approach and reduce the capabilities of pedestrians, to obtain a more realistic model.

The assumption of continuous and complete perception of global density information at current time is highly questionable in practical situations. Limited vision cones and restricted perception of global information comes through obstacles (walls, buildings), physical distance, visual orientation or the inability to see through a very dense crowd. Some effects of local vision on the behavior of crowds are obvious: in an evacuation scenario with two exit corridors, which cannot be seen 
from each other, pedestrians caught in a jam in front of one exit will not be able to see whether the other exit is free or also jammed.

These considerations motivated a new version of Hughes-type pedestrian dynamics based on localized perception of information, which we introduce in this section. The decision of each pedestrian is based on the perceived local density available in a limited domain, which can be e.g. interpreted as a vision cone. Concepts of limited cones have already been used in first-order models of collective behavior, see e.g. Ref. 22. Furthermore a local interaction mechanism between individuals as well as a smoothening kernel on the velocity field (to prevent unrealistic high frequency oscillations in the direction of motion) are incorporated. We begin with the detailed introduction of the macroscopic model and discuss the microscopic analogue thereafter.

\subsection{Macroscopic equations}

The starting point of our model is the assumption that pedestrians still perform the same path-optimization selection as in the Hughes' model, while the crowd state they act upon subjectively depends on their position and the amount of information they are able to perceive. Let $y \in \mathbb{R}^{2}$ be an auxiliary variable and $\phi(x, y): \mathbb{R}^{4} \rightarrow \mathbb{R}$ be a parametrized potential, such that $y \mapsto \phi\left(x_{0}, y\right)$ denotes the cost potential calculated by pedestrians located at $x_{0} \in \Omega$. To model space-dependent perception of information, suppose that for every $x$ the domain $\Omega$ decomposes into a visible subdomain $V_{x} \ni x$ and a hidden or invisible part $H_{x}=\Omega \backslash V_{x}$. We propose the following mechanism of restricted vision: if an area is visible, its density is known and priced accordingly in the path optimization. If however an area is not visible, its density is thought to be a constant $\rho_{H} \in \mathbb{R}_{0}^{+}$, which we assume to be uniform among all pedestrians. Exemplarily, setting $\rho_{H}=0$ implies that pedestrians assume that not visible areas to them are empty, hence they will have a strong incentive to explore unseen parts of the domain. On the contrary, pedestrians will avoid invisible areas when $\rho_{H} \approx \rho_{\max }$, as they assume high costs. An eikonal equation in $\Omega$ is hence solved in the auxiliary variable $y$ for every point $x$ as

$$
\left\|\nabla_{y} \phi(x, y)\right\|= \begin{cases}\frac{1}{f(\rho(y, t))}, & y \in V_{x}, \\ \frac{1}{f\left(\rho_{H}\right)}, & y \in H_{x},\end{cases}
$$

which gives the potential $\phi$ as function of two space variables. Note that this notion of local perception differs from other recent work, ${ }^{9}$ where a local average of the density is used. Each pedestrian uses the cost potential at her own position for the decision process. Computing $\nabla_{y} \phi(x, x)$ hence would, after normalization, give a new orientational vector field to be used in the unchanged transport equation. We however argue that it makes sense to include a notion of conviction to the model, which has previously not been considered. In order to do so, (3.1) 
is solved for every single exit. This results in the computation of $M$ potentials $\phi_{k}=\phi_{k}(x, t), k=1, \ldots, M$, which allows for cost comparison between exits, see Remark 3.2.

In regions of high density, decisions on the walking direction towards any of $k=$ $1, \ldots, M$ exits $\partial \Omega_{E_{k}}$ cannot arbitrarily deviate between neighbors. If a pedestrian prefers to walk against the direction of a predominant local flow, collision or friction losses in the movement will occur. Especially on the macroscopic level, in which we take an aggregate perspective, an incentive to change the flow cannot arise from one point in space alone. As we do not model the granular level of individual collision or friction, we propose the following mechanism:

(1) Each pedestrian carries an individual conviction strength $u(x)$ measuring its preference of its chosen exit over all others.

(2) There exists a local consensus process within the crowd, which results in the adjustment of the individual walking direction according to the predominant direction around them.

Hence, pedestrians adjust their own direction in order to prevail the flow rather than obstructing it. This can be seen as either a cognitive decision rule or a forced physical restriction. For a compactly supported interaction kernel $\mathcal{K}: \mathbb{R}^{2} \rightarrow \mathbb{R}$, we define the final walking direction $\varphi(x)$ at any point $x \in \Omega$ as

$$
\varphi=\frac{\rho u \star \mathcal{K}}{\rho \star \mathcal{K}}
$$

where the conviction $u(x)$ is given as

$$
u=\frac{\nabla_{y} \phi_{k^{\mathrm{opt}}}}{\left\|\nabla_{y} \phi_{k^{\mathrm{opt}}}\right\|}\left(\phi_{k^{\mathrm{opt}+1}}-\phi_{k^{\mathrm{opt}}}\right)
$$

obtained by comparing the cost potentials $\phi_{k}, k=1, \ldots, M$, associated to each of the exits:

$$
\begin{aligned}
k^{\mathrm{opt}}(x) & =\underset{k}{\operatorname{argmin}} \phi_{k}(x, x), \\
k^{\mathrm{opt}+1}(x) & =\underset{k \neq k^{\mathrm{opt}}}{\operatorname{argmin}} \phi_{k}(x, x) .
\end{aligned}
$$

Discontinuities in the velocity field due to the heterogeneity of decision making amongst pedestrians are hence partially compensated. To further smooth the model, we relax the strict restriction of $\|\nabla \varphi\|=1$ of Hughes' model and replace the normalization operator with a smooth approximation $\mathcal{P}: \mathbb{R}^{2} \rightarrow \mathbb{R}^{2}$ defined as:

$$
\mathcal{P}[x]:= \begin{cases}\frac{x}{\|x\|}, & \|x\|>\ell, \\ \sin \left(\frac{\pi}{2 \arctan (k \ell)} \arctan (k\|x\|)\right) \frac{x}{\|x\|}, & 0<\|x\| \leq \ell, \\ 0, & x=0,\end{cases}
$$




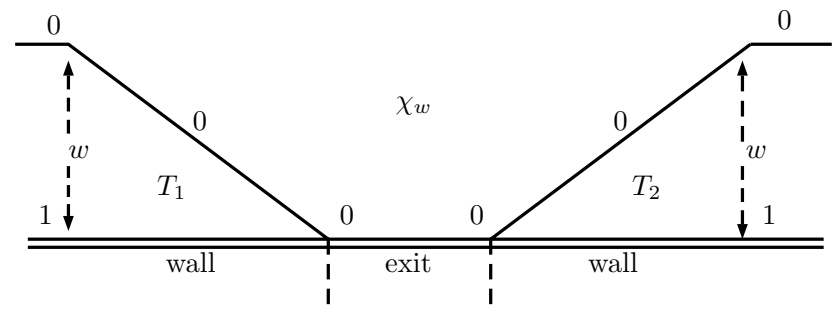

Fig. 1. Illustration of layer profile $\chi_{w}$ : wall and obstacle repulsion are embedded to the model with a fixed cost $W$ defined in terms of the layer profile $\chi_{w}(x) \in[0,1]$, see $(3.7)$, which indicates proximity to walls less than a width $w$, but vanishes away from obstacles and near exits to allow a proper outflow of pedestrians. $\chi_{w}$ equals one at walls and is the linear function given by vertex values within triangles $T_{1}, T_{2}$ near exits.

for some parameters $k, \ell>0$. We stress that this is not a technicality, as we here allow pedestrians to stop when being undecided. This is highly desirable from the modeling point of view, though on the other hand the modulus of the flux now is not a function of density alone, as one can see below.

Next we discuss the boundary conditions for the eikonal equations. Since we treat each exit separately, we set $\left.\phi_{k}\right|_{\partial \Omega_{E_{k}}}=0$ in the computation of $\phi_{k}$. No boundary conditions are imposed on the rest of the boundary $\partial \Omega_{\text {wall }}$.

Near-wall and near-obstacle effects have a strong influence on the dynamics on constrained macroscopic evolutions. We propose that pedestrians take into account walls and obstacles in their computation of optimal paths. Hence it is natural to include these effects as an additional fixed cost $W(x)$ on the right-hand side of the eikonal equation (3.1). We introduce a smooth layer profile $\chi_{w}(x) \in[0,1]$, which identifies areas close to walls but smoothly vanishes elsewhere and around exits to allow outflow. A typical choice of $\chi_{w}$ is illustrated in Fig. 1. For the sake of simplicity, we set

$$
W(x)=\frac{\chi_{w}(x)}{f\left(\rho_{\max }-\epsilon\right)},
$$

hence areas close to walls are penalized similar to high density areas.

Finally all terms are coupled to the continuity equation with velocity field $v(x, t)=-f(\rho) \mathcal{P}[\nabla \varphi]$ as in the original model. At exits, we prescribe a maximum outflow, given by $v(\xi, t)=-f(\rho) \mathbf{n}$ for all $\xi \in \partial \Omega_{\text {exit }}$. Taking all these considerations into account, the full macroscopic model reads as:

$$
\begin{gathered}
\partial_{t} \rho(x, t)+\nabla_{x} \cdot(-f(\rho(x, t)) \mathcal{P}[\nabla \varphi(x, t)] \rho(x, t))=0, \\
\varphi(x, t)=\frac{(\rho u \star \mathcal{K})(x, t)}{(\rho \star \mathcal{K})(x, t)}, \\
u(x, t)=\frac{\nabla_{y} \phi_{k^{\mathrm{opt}}}(x, x, t)}{\left\|\nabla_{y} \phi_{k^{\mathrm{opt}}}(x, x, t)\right\|}\left(\phi_{k^{\mathrm{opt}+1}}(x, x, t)-\phi_{k^{\mathrm{opt}}}(x, x, t)\right), \\
k^{\mathrm{opt}}(x, t)=\underset{k}{\operatorname{argmin}} \phi_{k}(x, x, t),
\end{gathered}
$$




$$
\begin{aligned}
& k^{\mathrm{opt}+1}(x, t)=\underset{k \neq k^{\mathrm{opt}}}{\operatorname{argmin}} \phi_{k}(x, x, t), \\
& \left\|\nabla_{y} \phi_{k}(x, y, t)\right\|= \begin{cases}\frac{1}{f(\rho(y, t))}+W(y), & y \in V_{x}, \\
\frac{1}{f\left(\rho_{H}\right)}, & y \in H_{x},\end{cases} \\
& \text { s.t. }\left.\quad \phi_{k}\right|_{\partial \Omega_{E_{k}}}=0, \quad k=1, \ldots, M, \forall t, \\
& \text { s.t. } \mathcal{P}[\varphi(\xi, t)]=\mathbf{n}, \quad \forall \xi \in \partial \Omega_{\text {exit }}, \quad \text { s.t. } \quad \rho(x, 0)=\rho_{0}(x) \text {. }
\end{aligned}
$$

We conclude the section with remarks on specific modeling assumptions.

Remark 3.1. (Vision cones) We have set aside formal statements regarding assumptions on the visible set $V_{x}$, but clearly we think of at least regular, connected and closed sets. A necessary condition is

$$
x \in V_{x}^{\circ}=\operatorname{int} V_{x},
$$

which implies that every pedestrian perceives some information from all directions. This restriction rules out e.g. angular vision cones (see Ref. 16) where pedestrians do not see what is happening behind them. In our model, (3.9) is necessary to exclude unrealistic situations where the chosen walking direction points outside the visible area. The inclusion of angular-dependent vision cones is certainly possible, but would imply a velocity-dependency and lead towards a second-order macroscopic model.

Remark 3.2. (Conviction term) The introduction of the conviction term $u(x)$ requires the computation of exit costs $\phi_{k}$ via the eikonal equation for individual exits, which appears to be a significant complication of the model. However, it is worth noting that the mechanism is almost identical to the original model. In Eq. (2.1b) the costs of walking towards any of the $K$ exits are compared, but only the minimal costs are used. Here, we simply store more information. This connection is also illustrated by looking at the numerical schemes for solving the eikonal equation: if a Fast Sweeping Method is used in e.g. a corridor with two exists, this essentially corresponds to solving for each exit separately if the minimization step is left out. If a Fast Marching Method is used, the conviction is directly related to the sequence in which vertices are promoted, with the least convinced vertex being assigned a cost the latest.

Remark 3.3. (Waiting behavior) The relaxation $\|\mathcal{P}[x]\| \leq 1$ implies that the modulus of the flux can be less than $f(\rho) \rho$ when pedestrians are undecided. This makes a rigorous analysis of the model equations a difficult task, which is not tackled in this work. The benefit of our formulation is that the problem of discontinuous velocity fields at sonic points has disappeared. Pedestrians at those hypersurfaces will not move unless the sonic points move. 


\subsection{The one-dimensional case}

Consider a one-dimensional corridor $\Omega=[0,1]$ with two exits and the uniform radial vision cone $V_{x}=[x-L / 2, x+L / 2] \cap \Omega$ of length $L$. Exit costs towards the left and right exit are computed at $y \in V_{x}$ as:

$$
\begin{aligned}
\phi_{L}(x, y, t) & =\int_{z<y, z \in H_{x}} \frac{1}{f\left(\rho_{H}\right)} d z+\int_{z<y, z \in V_{x}} \frac{1}{f(\rho(z, t))} d z, \\
\phi_{R}(x, y, t) & =\int_{z>y, z \in H_{x}} \frac{1}{f\left(\rho_{H}\right)} d z+\int_{z>y, z \in V_{x}} \frac{1}{f(\rho(z, t))} d z, \\
u(x, t) & =\phi_{L}(x, x, t)-\phi_{R}(x, x, t),
\end{aligned}
$$

as illustrated in Fig. 2. The cost potential $\phi$ is two-dimensional and $\partial_{y} \phi(x, y)$ gives the preferred walking direction that a pedestrian located at $x$ seeing $V_{x}$ assigns to $y \in[0,1]$. The walking directions chosen prior to the consensus process are hence given as $\partial_{y} \phi(x, x)$ along the diagonal of $[0,1]^{2}$. For every fixed $x \in[0,1]$, there is a unique sonic point $z(x)$, where $\phi_{L}(x, z(x))=\phi_{R}(x, z(x))$ and $\partial_{y} \phi(x, z(x))$ does not exist. As illustrated in Fig. 3, the individually preferred walking directions can switch multiple times between both exits, depending on the current density and the vision cones. At switching points, the preferred directions can point outwards (separation) or inwards (collision) and only the weighted interaction process (3.8b)(3.8c) generates a smooth velocity profile. In the Hughes' model, all vision cones are identical and there is a single separation point.

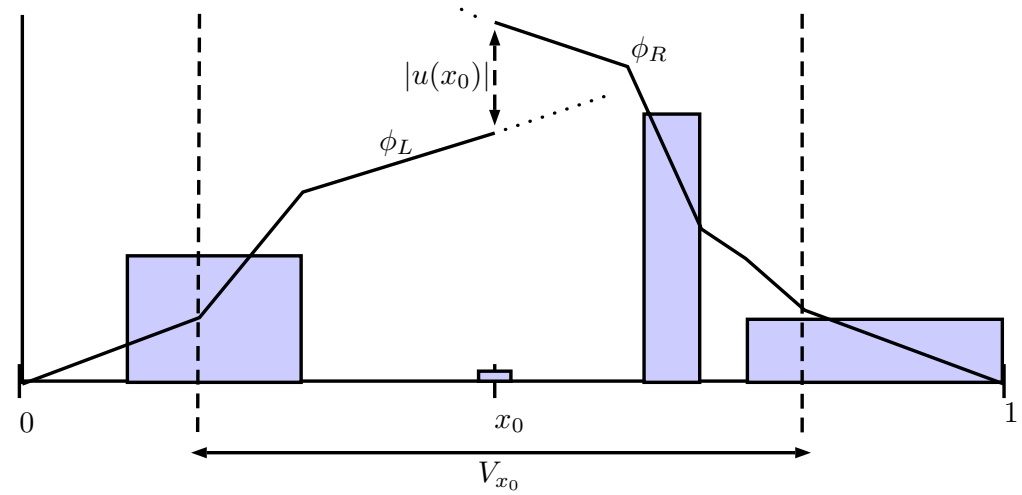

Fig. 2. Illustration of path optimization mechanism in 1D: a pedestrian located at $x_{0}$ computes and compares the cost potential $\phi_{L}, \phi_{R}$ of left vs. right exit in a corridor $[0,1]$. Next to its own negligible density, the present crowd consists of three blocks. Outside the vision cone $V_{x}$, the evacuation costs grow linearly at constant rate, as the local density is unknown. Within $V_{x}$, the slope of the cost potential increases with the pedestrian density. Preference is then given towards the exit with lower estimated cost. The conviction towards this decision is given as the cost benefit $\left|u\left(x_{0}\right)\right|=\left|\phi_{L}-\phi_{R}\right|$. 


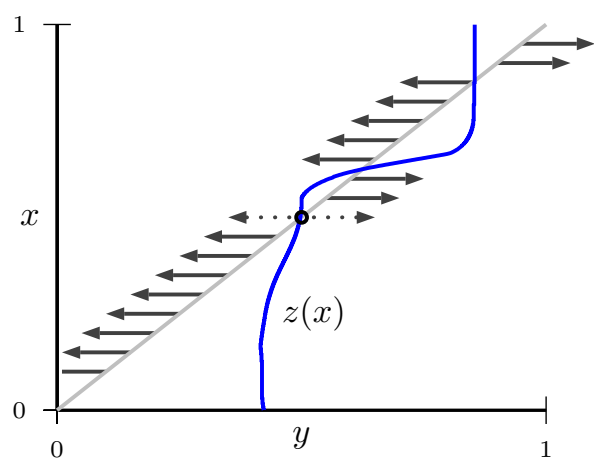

(a)

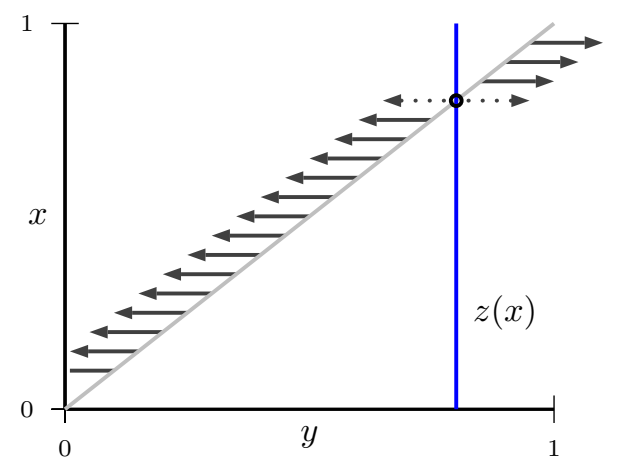

(b)

Fig. 3. Illustration of turning decisions of a 1D population for a given $\rho(x, t)$ : For every point $x$, we show the individual sonic point $z(x)$, where the costs $\phi_{L}(x, z(x))=\phi_{R}(x, z(x))$ coincide. The preferred walking direction for a pedestrian at $x$ is found along the diagonal $(x, x)$. If the sonic point is to the left, the pedestrians aim to walk towards the right and vice versa. No direction is preferred and the conviction is zero if the curve of sonic points intersects the diagonal. (a) Local vision cones: the preferred direction alters and creates multiple points of separation and collision. The resulting velocity field is obtained by the smoothening interaction process (3.8b)-(3.8c). (b) Hughes' model: all vision cones coincide, hence there is one identical sonic point common to all pedestrians.

\subsection{Microscopic interpretation}

We conclude this section by briefly commenting on the modeling of local vision at the microscopic level. The microscopic modification is straightforward and uses the same ideas as at the macroscopic level. It corresponds to updating the position $X=X(t)$ according to a potential which depends on local information only. Its calculation is based on the same equations as in the macroscopic model (3.8f) but using the smoothed empirical density $\rho_{g}^{N}$ instead of $\rho$. The position update is based on Eqs. (3.8b)-(3.8e). Hence individuals choose the path towards the exit with the lowest cost, but weigh their decision according to the predominant direction chosen around them. For further details on the implementation we refer to Algorithm 2 presented in Sec. 4.

\subsection{Analysis of the domains of dependence}

In this subsection, we will discuss some mathematical properties of the solutions of the eikonal equation (3.8f). From the construction of the model, the potential $\phi(x, y, t)$ has to be computed for every $x \in \Omega$ on the entire domain $\Omega$, which counterbalances the idea of locality and increases the computational cost considerably. We show here that the computation of the potential can actually be reduced to a subset of $\Omega$, called the effective domain of dependence, for every $x$. Only this subset, which contains $V_{x}$, is considered in the individual local planning problem and corresponds to the reduction of the computational cost. 
The following proofs rely crucially on the optimal path property of the characteristics associated to the eikonal Eq. (3.8f). We recall ${ }^{26,5}$ that by Fermat's principle the characteristic paths associated to $\phi(x, y, t)$, given by the solution of:

$$
\gamma_{x, t}^{z}(s) \subset \Omega: \gamma(0)=z, \quad \dot{\gamma}(s)=-\nabla \phi(x, \gamma(s), t) \quad \text { for all } s \geq 0
$$

are the optimal paths for the cost defined as

$$
c(y, t)= \begin{cases}\frac{1}{f(\rho(y, t))}+W(y), & y \in V_{x}, \\ \frac{1}{f\left(\rho_{H}\right)}, & y \in H_{x} .\end{cases}
$$

Moreover, the potential is the value function for that cost. Hence it is decreasing along these paths and satisfies the optimality condition

$$
\phi\left(x, \gamma_{x, t}^{z}(a), t\right)-\phi\left(x, \gamma_{x, t}^{z}(b), t\right)=\int_{a}^{b} c\left(\gamma_{x, t}^{z}(s), t\right) d s, \quad \text { for all } 0 \leq a<b
$$

being zero at its corresponding exit $\partial \Omega_{\text {exit }}$. Furthermore, the curves $\gamma_{x, t}^{z}$ are the optimal paths to achieve the exit, i.e. they verify the following global optimality condition

$$
\phi(x, z, t)=\int_{0}^{T_{z}} c\left(\gamma_{x, t}^{z}(s), t\right) d s \leq \int_{0}^{\tilde{T}_{z}} c(\tilde{\gamma}(s), t) d s
$$

for all $\tilde{\gamma}$ curves joining $z$ to any point in the exit $\partial \Omega_{\text {exit }}$, where $T_{z}$ is the optimal time to achieve the exit for the point $z \in \Omega$ and $\tilde{T}_{z}$ is the time to achieve the exit for the path $\tilde{\gamma}$.

Lemma 3.1. Consider any fixed $V_{x} \subset \Omega$ and that $f(\rho)>0,0 \leq \rho<\rho_{\max }$. Let $\phi_{H}$ be the global solution of the eikonal equation $\left\|\nabla \phi_{H}\right\|=1 / f\left(\rho_{H}\right), \phi_{H}(x)=0$ on $\partial \Omega_{\text {exit }}$. Define the minimum of $\phi_{H}$ in $V_{x}$ as

$$
m_{H}:=\min _{z \in V_{x}} \phi_{H}(z)
$$

and the corresponding superlevel set of $\phi_{H}$ as

$$
M_{H}:=\left\{x \in \Omega: \phi_{H}(x) \geq m_{H}\right\} .
$$

Then the problem of computing the local potential $\phi(x, y, t)=: \tilde{\phi}(y)$ out of $(3.8 \mathrm{f})$ on $\Omega$ reduces to the following problem on $M_{H}$ :

$$
\begin{cases}\left\|\nabla_{y} \tilde{\phi}(y)\right\|=\frac{1}{f(\rho(y, t))}+W(y), & \text { in } V_{x}, \\ \left\|\nabla_{y} \tilde{\phi}(y)\right\|=\frac{1}{f\left(\rho_{H}\right)}, & \text { in } M_{H} \backslash V_{x}, \\ \tilde{\phi}(y)=m_{H}, & \text { on } \partial M_{H} \backslash \partial \Omega_{\mathrm{wall}} \text { (B.C.). }\end{cases}
$$


Proof. If an exit is visible then $m_{H}=0, M_{H}=\Omega$ and the assertion is trivial. If no exit is visible then by construction $V_{x} \subset M_{H}$ and $\phi_{H}=m_{H}>0$ on $\partial M_{H}$. As the walking costs are always positive, $c(y, t)>0$, we get $\phi(x, y, t)>m_{H}$ for all $y \in \operatorname{int} M_{H}$. On the other hand, any point $z \in \Omega \backslash M_{H}$ satisfies $\phi(x, z, t)<m_{H}$ and hence $\gamma_{x, t}^{z}(s)$ does not intersect $V_{x}$, otherwise the cost should be larger at a middle point than initially, which would contradict the optimality of the path $\gamma_{x, t}^{z}(s)$ in (3.11). Hence $\partial M_{H}$ is the maximal level set consisting of points whose optimal paths do not cross $V_{x}$, and therefore, $\phi(x, z, t)$ can be computed from (3.8f) with constant right-hand side outside $M_{H}$.

Definition 3.1. Consider a fixed visibility area $V_{x}$. For a $z \in \Omega$, denote the default optimal path $\gamma_{H}^{z}$ as the parametrized curve associated to a gradient walk along $\phi_{H}$ starting in $z$, that is

$$
\gamma_{H}^{z}(s) \subset \Omega: \gamma(0)=z, \quad \dot{\gamma}(s)=-\nabla \phi_{H}(\gamma(s)) \quad \forall s \geq 0 .
$$

Next, define the characteristics' shadow $V^{\#}$ as the set of all points, whose default optimal path crosses the visibility area, hence

$$
V^{\#}:=\left\{z \in \Omega: \gamma_{H}^{z} \cap \operatorname{int} V_{x} \neq \emptyset\right\} .
$$

Note that $V^{\#} \subset M_{H}$ since any default optimal path outside of $M_{H}$ cannot intersect with $V_{x}$ as proven in the previous lemma.

Lemma 3.2. Consider any fixed $V_{x} \subset \Omega$ and assume that $f(\rho)>0$ is increasing in $0 \leq \rho<\rho_{\max }$, with $\rho_{H}=0$, then the problem of computing the local potential $\tilde{\phi}(y)$ out of (3.1) further reduces to the following problem on $V^{\#}$ :

$$
\begin{cases}\left\|\nabla_{y} \tilde{\phi}(y)\right\|=\frac{1}{f(\rho(y, t))}+W(y), & \text { in } V_{x}, \\ \left\|\nabla_{y} \tilde{\phi}(y)\right\|=\frac{1}{f(0)}, & \text { in } V^{\# \backslash V_{x},} \\ \tilde{\phi} \equiv \phi_{H}, & \text { on } \partial V^{\#} .\end{cases}
$$

Proof. For any point $z$ whose default optimal path $\gamma_{H}^{z}$ that does not intersect with $V$, the claim is that $\tilde{\phi}(z)=\phi_{H}(z)$ due the monotonicity of the cost function, i.e.

$$
\frac{1}{f(\rho(y, t))}+W(y) \geq \frac{1}{f(0)} .
$$

To prove this, let us denote by $T_{z}^{H}$ the optimal time to get to the exit for the default optimal path $\gamma_{H}^{z}$.

We first take $\gamma_{x, t}^{z}(s)$ as a candidate path in the global optimality condition (3.12) for the eikonal equation with right-hand side $c_{H}=\frac{1}{f(0)}$. Being $\gamma_{x, t}^{z}(s)$ a path joining 


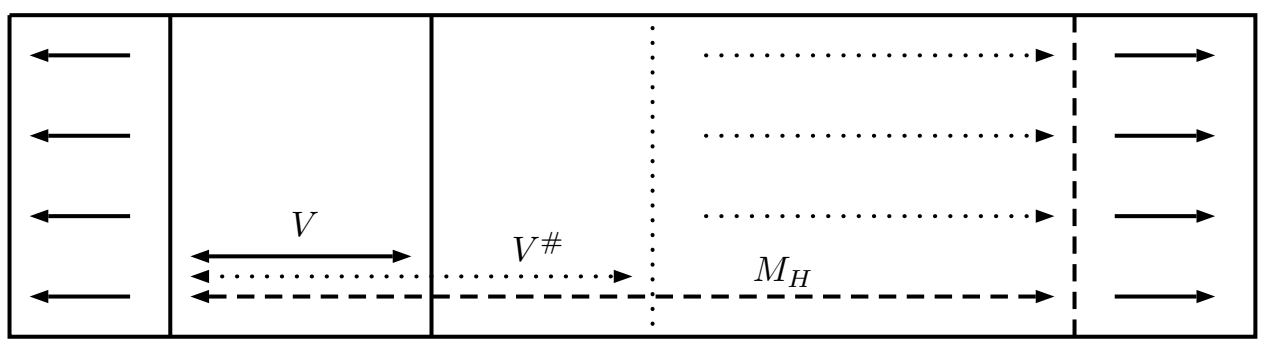

Fig. 4. Illustration of the domains $V, M_{H}$ and $V^{\#}$ for computation of the visibility area potential $\tilde{\phi}$ for the case of a corridor with two opposing exits: the problem on $\Omega$ generally reduces to a HJequation on $M_{H}$, as by construction $\tilde{\phi}$ coincides with $\phi_{H}$ outside of $M_{H}$ (Lemma (3.1), $-\nabla \phi_{H}$ solid arrows). If $\rho_{H}=0$, any default optimal path of $\phi_{H}$ that does not intersect $V$ remains optimal, as indicated by dotted arrows, and the problem reduces to $V^{\#}$ (Lemma 3.2).

$z$ to a point in the exit and $\gamma_{H}^{z}(s)$ the optimal one, we conclude

$$
\begin{aligned}
\phi_{H}(z) & =T_{z}^{H} c_{H} \leq T_{z} c_{H} \\
& \leq \int_{0}^{T_{z}} c\left(\gamma_{x, t}^{z}(s), t\right) d s=\tilde{\phi}(z) .
\end{aligned}
$$

Now, we take $\gamma_{H}(z)$ as a candidate path in the global optimality condition (3.12) for the eikonal equation with right-hand side $c(y, t)$. It is an admissible path as it connects $z$ to a point at the exit and the cost along its path coincides with $c_{H}=c\left(\gamma_{H}^{z}(s), t\right)$ for all $s \in\left[0, T_{Z}^{H}\right]$ since the path does not cross $V$. Then, we get

$$
\tilde{\phi}(z) \leq \int_{0}^{T_{z}^{H}} c\left(\gamma_{H}^{z}(s), t\right) d s=T_{z}^{H} c_{H}=\phi_{H}(z),
$$

leading to the stated result.

We illustrate Lemmas 3.1 and 3.2 in Fig. 4. It can be seen that the reduction of the computational domain from $M_{H}$ to $V^{\#}$ can be significant, as the size of $M_{H}$ depends on the closeness of $V$ to the nearest exit, not on the size of $V$. For the exemplary geometry of Fig. 4, the boundary of $V^{\#}$ coincides with the sonic points of $\phi_{H}$, but this is not true in general. Furthermore, it is easy to see why the computational domain cannot be reduced further. Suppose that $\rho(\cdot, t)$ is spatially homogeneous, then $-\nabla \phi$ in $V^{\#} \backslash V$ points to the left exit as in the eikonal case. On the other side, one can choose a situation with a large density at the left boundary of $V$ that leads to right-pointing $-\nabla \phi$ in $V^{\#} \backslash V$.

\section{Computational Methods}

In this section we present a microscopic and a macroscopic numerical solver to simulate the classic and the local version of the Hughes model. The proposed methods 
have been implemented on regular and triangular meshes in 2D to allow for flexible discretizations of polygonal domains with one or several obstacles.

For the macroscopic system (3.8) we use the following explicit iterative algorithm:

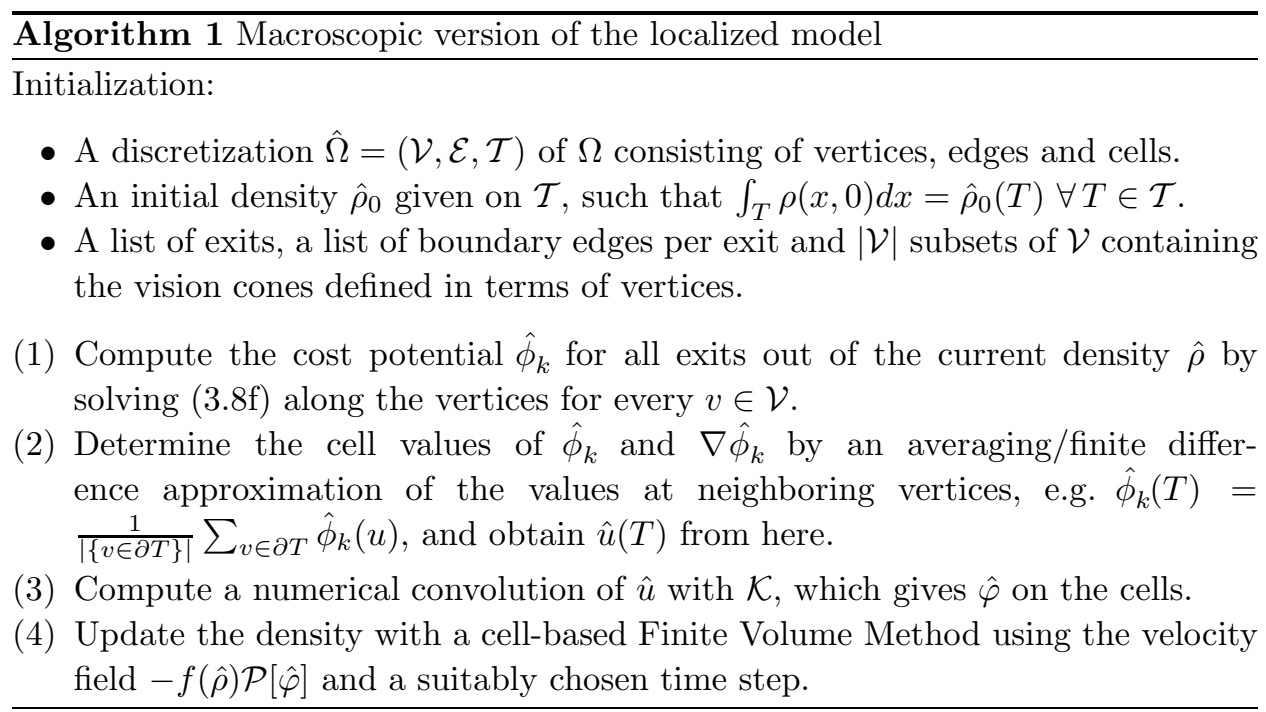

The discretization is either a regular grid or an unstructured regular triangular mesh to allow more complex geometries. For solving the eikonal equations, one can choose between Fast Sweeping Methods ${ }^{40,34}$ and Fast Marching Methods. ${ }^{30,36}$ The former is based on a Gauss-Seidel iteration, which updates the solution by passing through the computational domain in alternate pre-defined sweeping directions. A rectangular grid provides a natural ordering of all grid points. This ordering does not exist on an unstructured grid and is replaced by a general ordering strategy by introducing reference points, which is done once. Then the solution at each node is consecutively updated by running through the ordered lists. Marching methods update vertices in a monotone increasing order, where in every iteration a list of candidate values is available by finite difference approximation from previously approved values. The smallest value of all of candidate values is then promoted and assigned to its vertex.

As a Finite Volume Method we use the first-order monotone FORCE scheme. ${ }^{37,20}$ Some post-processing between the steps of Algorithm 1 is required: outward-pointing components of $\nabla \hat{\phi}_{k}$ are removed along the boundary, suitable values of $\nabla \hat{\phi}_{k}$ are ensured at corners of $\Omega$, and the max outflow condition (3.8h) is enforced at cells neighboring exit edges. 
The analogous algorithm used for the numerical simulation of the microscopic model is as follows:

Algorithm 2 Microscopic version of the localized model

Let us consider a system of $N$ particles, which are initially located at positions $X^{j}(0)=X_{0}^{j}$. In every time step $t^{i}=i \Delta t$ we update the particle position as follows:

(1) Determine the empirical density at time $t^{i}$ :

$$
\rho_{g}^{N}\left(x, t^{i}\right)=\frac{1}{N} \sum_{j=1}^{N} g\left(x-X^{j}\left(t^{i}\right)\right),
$$

where $g$ denotes a Gaussian.

(2) Solve the eikonal equation to determine the weighted distance to each exit $\phi^{k}=\phi^{k}\left(x, t^{i}\right), k=1, \ldots, M$ :

$$
\begin{aligned}
\left\|\nabla \phi^{k}\left(x, y, t^{i}\right)\right\| & = \begin{cases}\frac{1}{f\left(\rho_{g}^{N}\left(y, t^{i}\right)\right)}+W(y), & \text { if } y \in V_{x}, \\
\frac{1}{f\left(\rho_{H}\right)}, & \text { otherwise. }\end{cases} \\
\phi^{k}\left(x, t^{i}\right) & =0 .
\end{aligned}
$$

(3) Update the position of each particle $X^{j}$ via:

$$
\left.\dot{X}^{j}\left(t^{i}\right)=-f^{2}\left(\rho_{g}^{N}\left(x, t^{i}\right) \cdot \nabla \varphi\left(x, t^{i}\right)\right)\right),
$$

where $\varphi\left(x, t^{i}\right)$ is determined by $(3.8 \mathrm{~b})$.

\section{Results}

In this section we illustrate the dynamics of the localized model for crowd dynamics with examples in one and two dimensions. In all simulations we consider an evacuation scenario of a corridor, where a given initial distribution of people tries to leave the rectangular domain through either one of the two exits as fast as possible. We compare the evacuation time, i.e. the time at which all individuals have left the domain, with respect to different parameters, e.g. vision cones. In the case of a global vision cone we obtain Hughes'-type dynamics. As a flux law, we choose to the LWR function

$$
f(\rho)=\rho(1-\rho),
$$

setting $\rho_{\max }=1$ throughout this section.

\subsection{D corridor - Macroscopic model}

In our first example the domain $\Omega$ corresponds to the unit interval $\Omega=[0,1]$ with two exits located at either end, i.e. at $x=0$ and $x=1$. We consider an evacuation 
scenario in which two groups, one of them being densely packed, want to leave through either one of the exits:

$$
\rho_{0}(x)= \begin{cases}0.85 & 0 \geq x \geq 0.3, \\ 0 & 0.3<x<0.6, \\ 0.25 & 0.6 \geq x \geq 1,\end{cases}
$$

and we set the width of the vision cone to $L=0.75$. The resulting dynamics are illustrated at four time steps in Fig. 5. Within the left block, some pedestrians decide to walk towards the right exit, as they are aware of the high density on their left and account for a higher walking cost compared to the relatively empty right-hand side. After the separation the right-moving part evolves as a rarefaction wave, as known from the LWR model. As the distance between the wave and the

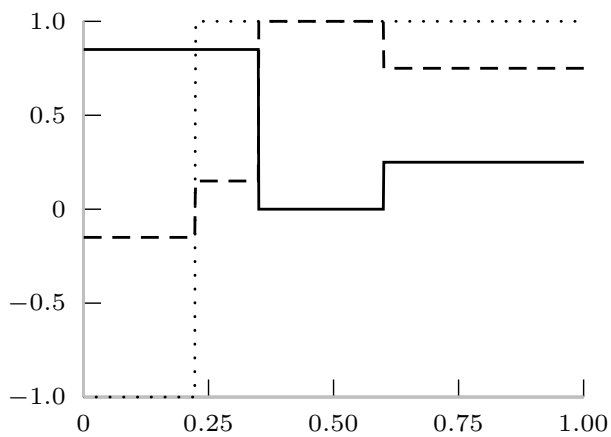

(a) $t_{0}=0$

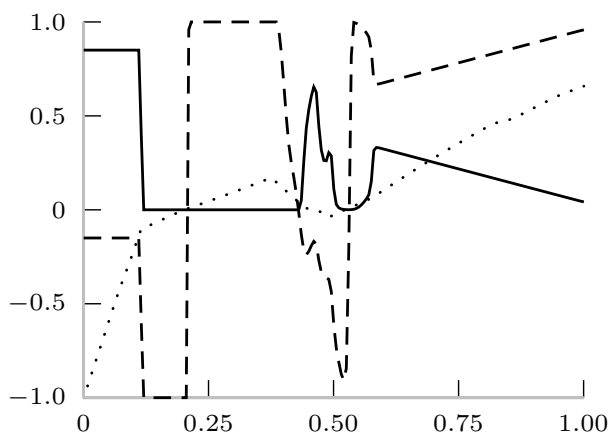

(c) $t_{2}=0.71$

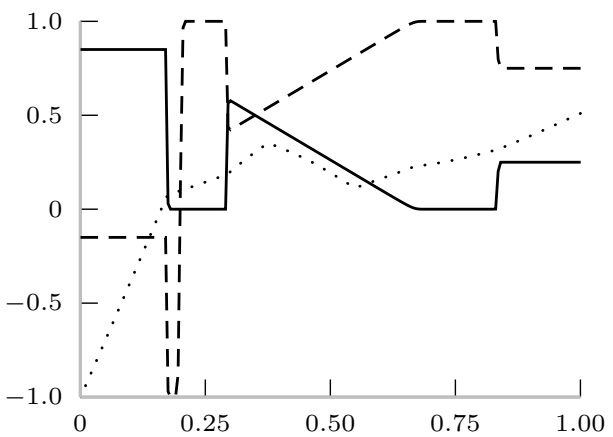

(b) $t_{1}=0.31$

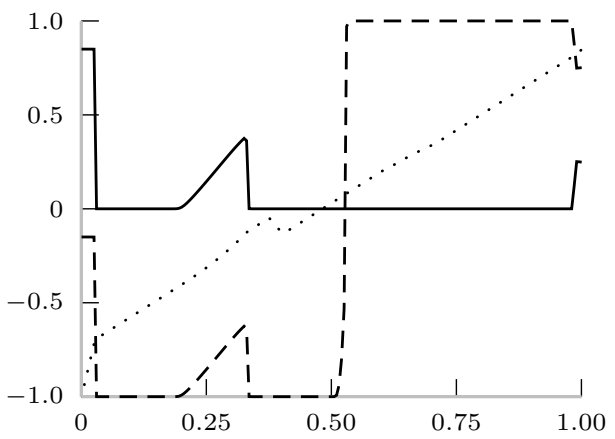

(d) $t_{3}=1.29$

Fig. 5. Exemplary evolution of the 1D model showcasing a turnaround behavior due to localized perception of information [density $\rho$ solid $(-)$, speed $v=-f(\rho) \mathcal{P}[\nabla \varphi]$ dashed (--) and directional conviction $\phi_{R}-\phi_{L}$ dotted (..)]. (a) Piecewise constant initial density. Part of the left crowd initially decides to move right in order to avoid the high density jam. (b) The separated block moves to the right in a rarefaction-wave manner. (c) The wave is again separated as the high density jam gets out of sight for centrally located pedestrians, who hence prefer the left exit and turn. (d) The turnaround is complete and remaining pedestrians will exit on the left. 
left-moving shock grows, the effects of the local vision cone become apparent. At some point pedestrians moving to the right do not see the high density at the left exit anymore and start to turn around. Therefore the rarefaction wave splits again - one part continues while the other one turns around and moves back to the left exit. The turnaround occurs in several stages:

(1) A new sonic point arises, where pedestrians are undecided between both exits. The walking direction is unchanged as the local consensus process (3.8b) prevents an immediate switching.

(2) When a critical mass of density and conviction opting for walking to the left, the velocity after consensus switches continuously and passing through zero. This creates a temporary collision point, as there are still pedestrians to the left of the sonic point which walk towards the right.

(3) The density at the collision point increases, which causes pedestrians to the left of the collision point to turn around too, as a higher density is in their way, as it can be seen in Fig. 5(c).

(4) Finally, all pedestrians to the left of the initial sonic point have turned and walk towards the left (Fig. 5(d)).

This new behavioral pattern is entirely consistent with the idea of constant re-evaluation of the optimal path based on restricted information and cannot be observed in the original Hughes' model. We note that without the smoothening properties of the model around points of equal costs one obtains strong oscillations in the turning behavior, which causes severe numerical problems. The exact parameters of the simulation can be found in Appendix A.1

\subsection{D corridor - Microscopic model}

We illustrate the dynamics of the microscopic model in a two-dimensional symmetric corridor $\Omega=[0,1] \times\left[0, \frac{1}{2}\right]$ with exits at the left and right side, i.e. $x=0$ and $x=1$. The $1 \mathrm{D}$ case of Sec. 5.1 can be interpreted as a projection of this two-dimensional geometry. We consider the same initial distribution of individuals, i.e. the positions of all 500 particles are distributed according to the initial pedestrian density (5.2). For $L=0.25$, Figs. 6(a)-6(f) nicely illustrates a similar turnaround behavior as in the $1 \mathrm{D}$ macroscopic simulations. At the beginning the group close to the left exit splits, one part exits through the left exit and the other one moves towards the more distant right exit. As the density close to the left exit decreases in time, the group moving towards the more distant exit splits again, i.e. parts of the group turn around and move back again. We marked all individuals, which initially moved towards the right but then turn around, with red triangles. Furthermore, Fig. 6(g) shows the change of the evacuation performance for different sizes of the local vision cones $L$. Here we plot the percentage of the total initial mass outside the domain versus time. Decreasing $L$ and hence the perceived information, 


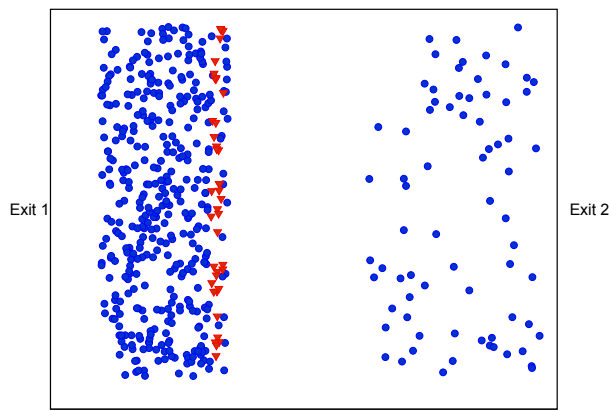

(a) Time $t=0$

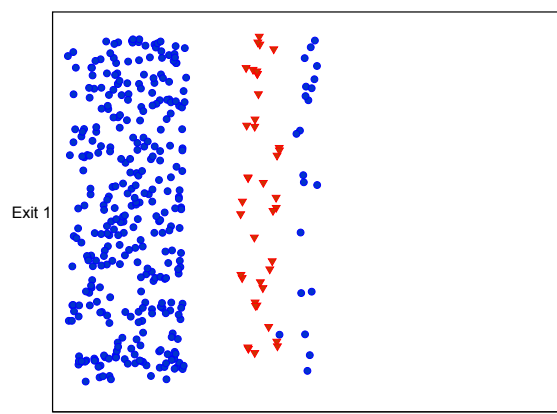

(c) Time $t=0.4$

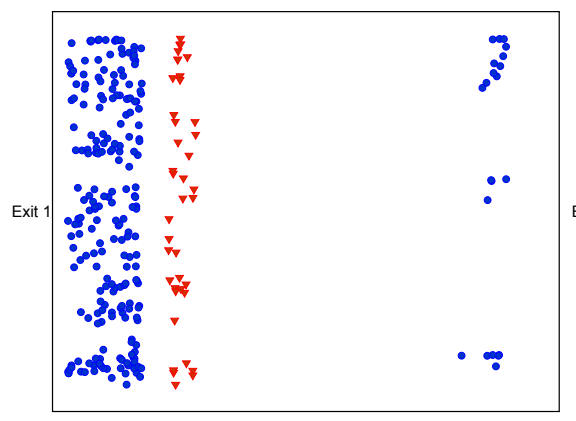

(e) Time $t=0.8$

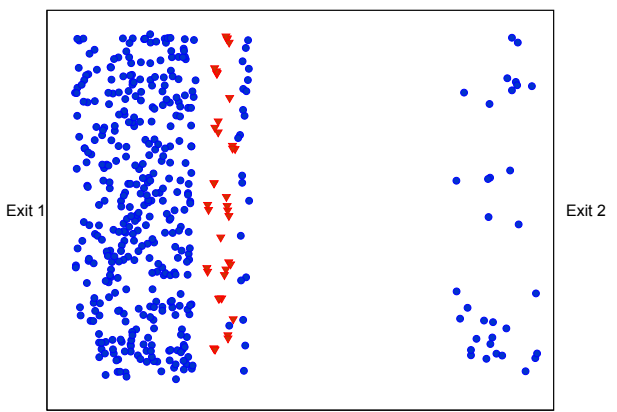

(b) Time $t=0.2$

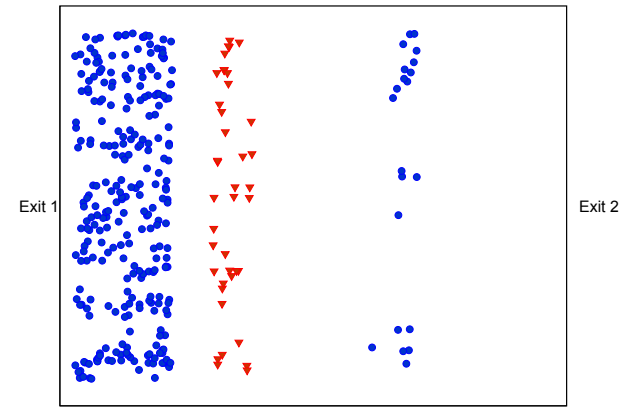

(d) Time $t=0.6$

Fig. 6. (Color online) Dynamics of the microscopic model in a two-dimensional corridor with two exits at the right and left. We observe a similar behavior as in the 1D simulation in Sec. 5.1 individuals (visualized by red triangles) initially decide to move to the more distant exit, but after the congestion at the resolves in time, they turn around and take the closer exit. (a)-(f): particle solution for different times and $L=0.25$, (g): exit percentage over time for different values of $L$. 


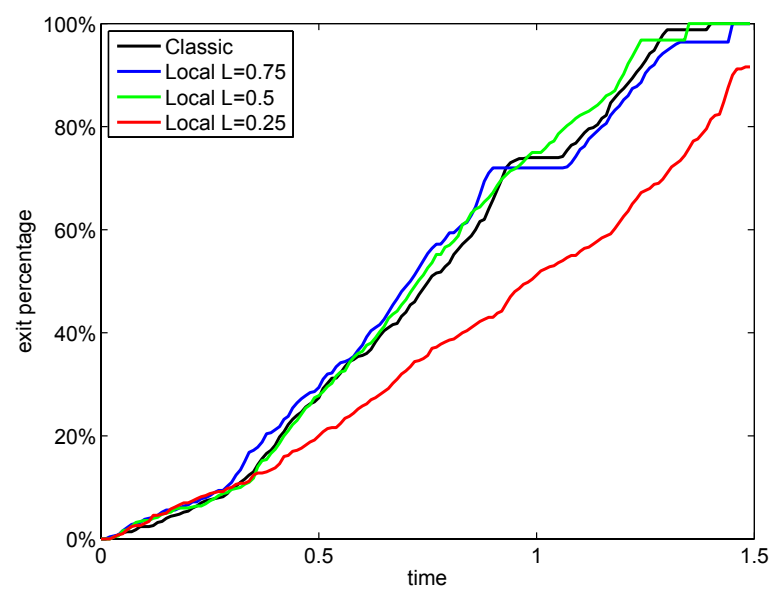

(g) Exit percentage

Fig. 6. (Continued)

we observe that the overall evacuation performance first is merely diminished, and only begins to drop significantly after a certain threshold. The evacuation time will approach the uninformed eikonal case $L=0$, which is not shown. All parameters can be found in Appendix A.2.

\subsection{D nonsymmetric corridor - Macroscopic model}

Now we turn to the macroscopic model in two dimensions. Again we consider the corridor $\Omega=[0,1] \times\left[0, \frac{1}{2}\right]$, the exits however form only a part of the left and right edges, hence we obtain a fully two-dimensional dynamics where boundary conditions matter. The left exit is located between $(0,0)$ and $(0,0.1)$ and the right exit is the segment connecting $\left(1, \frac{1}{2}\right)$ and $(1,0.4)$. The initial density Fig. $7(\mathrm{a})$ is given as a low density group of pedestrians on the left and a high density group on the right:

$$
\rho_{0}(x, y)= \begin{cases}0.1 & 0.05 \leq x \leq 0.3, \quad 0 \leq y \leq 0.25 \\ 0.95 & 0.6 \leq x \leq 0.95 \\ 0 & \text { otherwise }\end{cases}
$$

We first study the case of global vision $L=\infty \Leftrightarrow V_{x}=\Omega$ in Fig. 7. In (b), the low density group turns towards left and is quickly vacated. The high density group on the other side splits along a curve of sonic points. Pedestrians turning to the right cause a jam in front of the right exit, whereas left-turning pedestrians occupy the corridor in a rarefaction-type manner inherited by the physical flux law (5.1). Upon arrival at the left exit, pedestrians pile up and form a new jam (c). Hence, a fraction of the density turns around again and heads for the right exit ((d), around 


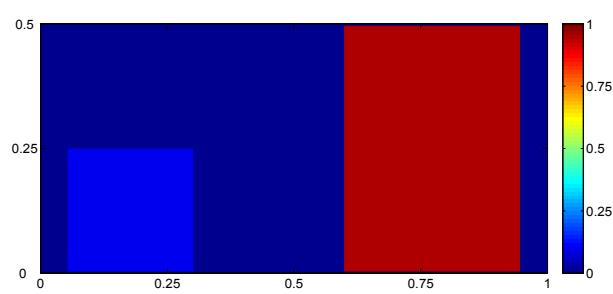

(a) Time $t=0$

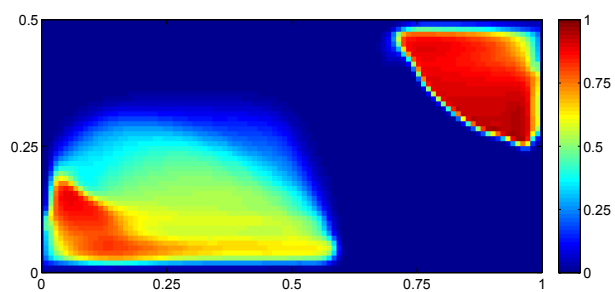

(c) Time $t=1.07$

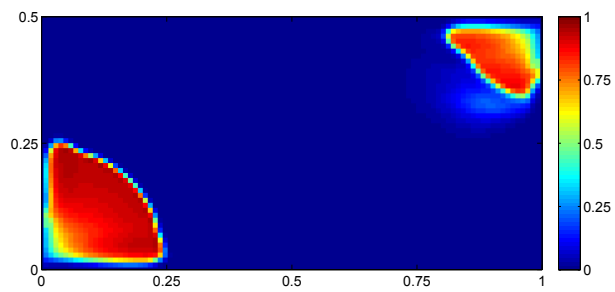

(e) Time $t=2.1$

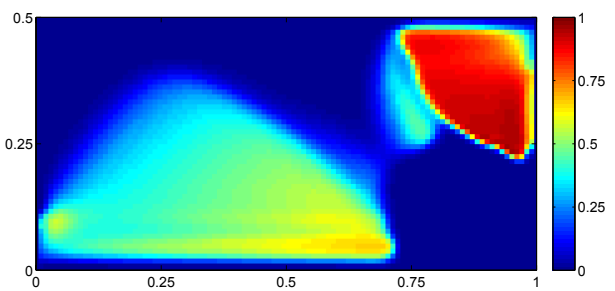

(b) Time $t=0.8$

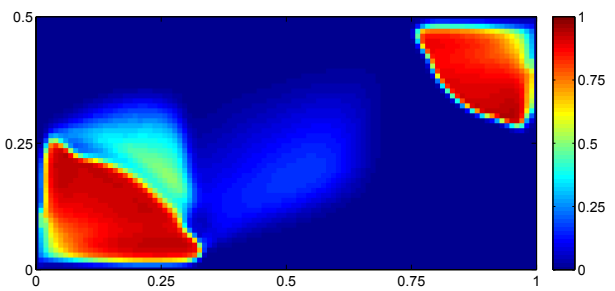

(d) Time $t=1.4$

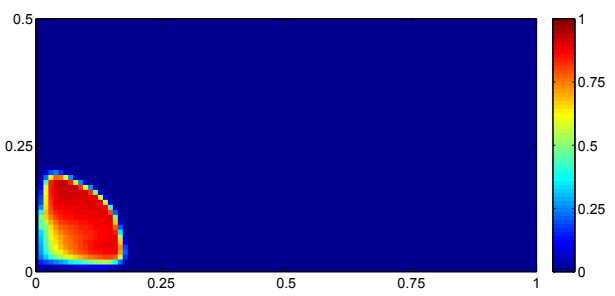

(f) Time $t=2.75$

Fig. 7. Two-dimensional macroscopic dynamics: we simulate model (3.8) with global visual perception $V_{x}=\Omega$. Two groups of pedestrians are initially placed in a corridor with a lower left and an upper right exit. The high density group separates according to the path optimization mechanism, as illustrated in several time snapshots of the density in (a)-(f). The right exit is vacated before the left exit and the final evacuation time (not shown here) is $\approx 3.4$.

$(0.5,0.25))$, having to cross most of the corridor again (e). However, most of the pedestrians are committed to the left exit and do not turn, because the severeness of the left jam does not compensate their expected travel time, and the left exit is vacated later than the right exit (f).

Compared to the classical Hughes model, the relaxation term (3.6) and the conviction-based interaction (3.8b)-(3.8c) allow for a smooth turning behavior. The wall-repulsion (3.7) causes a density gap, which is different to zero-flux conditions as in Ref. 28, but prevents any spurious effects of the boundary flux.

It is clear that even the planning algorithm incorporated into the classic Hughes' model does not lead to an optimal evacuation. One reason for suboptimality in Fig. 7 is that pedestrians have to keep in motion constantly but cannot predict the 


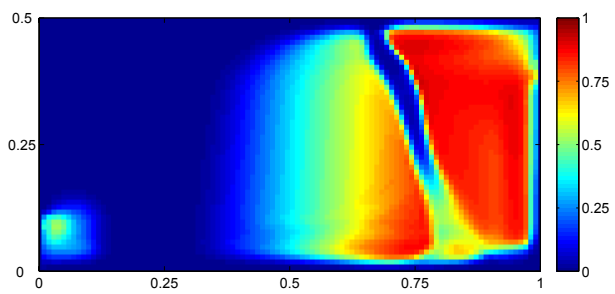

(a) Time $t=0.25$

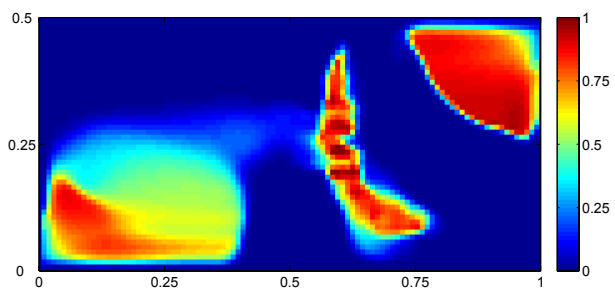

(c) Time $t=1.07$

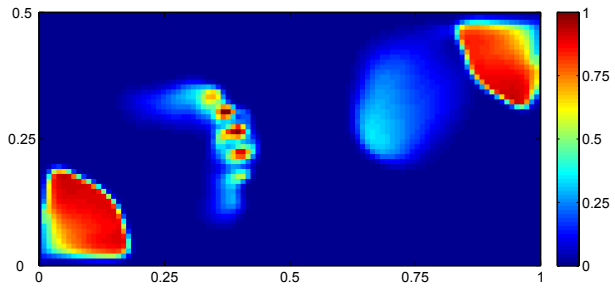

(e) Time $t=2.1$

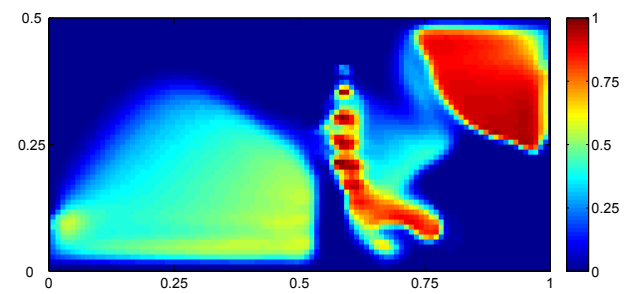

(b) Time $t=0.8$

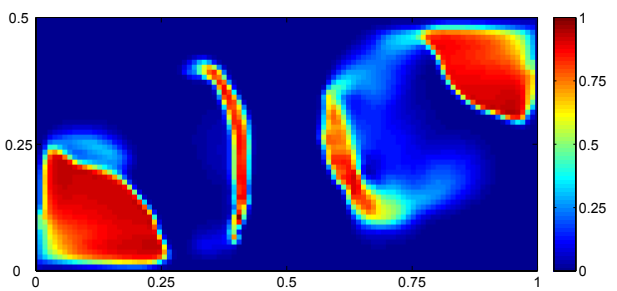

(d) Time $t=1.4$

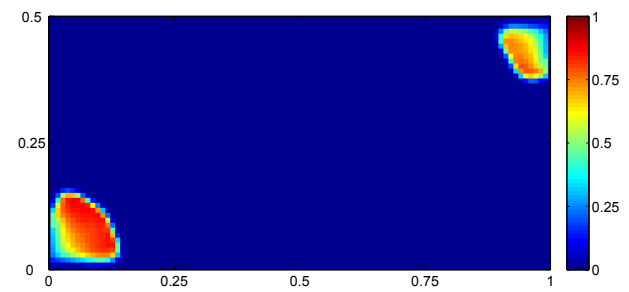

(f) Time $t=2.75$

Fig. 8. Two-dimensional macroscopic dynamics: we simulate model (3.8) with local visual perception $L=0.75$ and the same initial configuration as in Fig. 7. In the time snapshots of the density, we observe again an initial separation of the high density group (a). A waiting phenomena of up to two local groups is clearly observed (b)-(d). Crucially, waiting pedestrians are able to choose their exit later (d)-(e), which leads to a rather simultaneously clearing of both jams (f), as opposed to Fig. 7 . The final evacuation time (not shown here) is $\approx 3.025$.

occurrence of future jams. Hence, pedestrians are likely to walk towards an exit that will be blocked in the future, as seen in the example.

In Fig. 8 we study the same initial configurations with localized perception and a radial vision cone of diameter $L=0.75$. The initial separation phase (a) is similar to Fig. 7. As pedestrians move from the right to the left, the right jam gets out of sight and its influence diminishes. At the same time, the density on the left becomes visible. At a certain point a balance is achieved and pedestrians locally accumulate around an area of equal walking costs, where in this case they are able to stop (b)-(c). Hence, we observe a waiting behavior which cannot be observed in classical Hughes' type models. Looking from (c) to (d), a high density jam forms at the left exit, which causes part of the left-walking pedestrians to turn right 


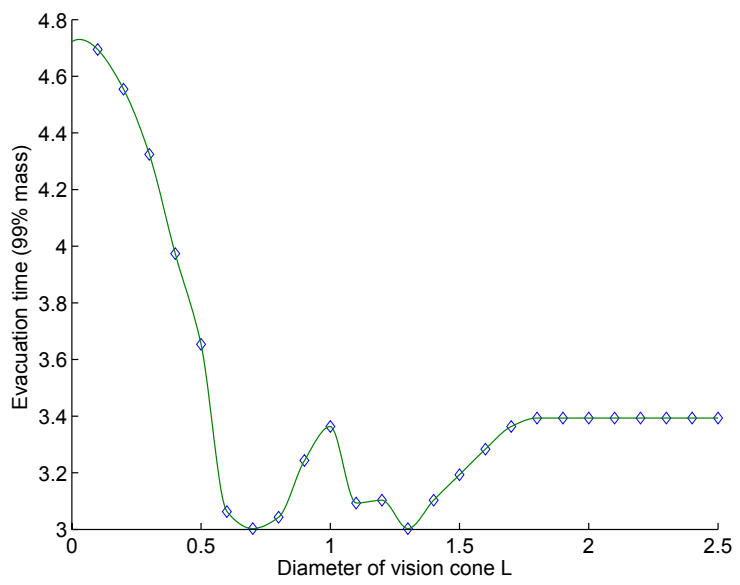

Fig. 9. Evacuation time of the macroscopic model for varying vision cone diameters $L$ : the performance can improve with limited vision. $L=0$ corresponds to the eikonal case whereas $L>2.5$ implies unlimited vision in the given corridor.

after enough conviction is gathered. Together with some outflow of the first waiting group, a second waiting group is formed (d). Pedestrians in a waiting group choose to move if one direction becomes favorable. As both jams at the exits reduce at the same rate, the left waiting group walks to the left and vice versa (e). Finally, the waiting groups dissolve and the exits get vacated at a rather similar time (f), and the evacuation time improves compared to Fig. 7 .

The fact that evacuation performance can improve under limited perception of information is surprising at first glance. Our simulations give a good explanation for the phenomena: as pedestrians show a waiting behavior, they are less likely to be trapped in the jam arising at the left exit. In fact, the waiting is made possible by the combined effect of multiple sonic points due to local vision and the smoothed turning mechanism. Naturally, this is not generally the case and cannot be a priori answered. For small vision lengths $L$, the dynamics will converge to the velocity field given by the eikonal equation, which is our initial configuration will exit almost all pedestrians using the right exit and perform poorly. In Fig. 9, we study the evacuation time of $99 \%$ of the initial mass as a function of the diameter $L$. We unexpectedly find in this case two optimal values of $L$ for which the evacuation time is minimal. The classical Hughes' evacuation time ( $L$ large) is always less or equal than the eikonal case $(L=0)$, however there is no way to generally argue that there will always be a minimum in-between.

\section{Conclusion}

In this work we introduced a localized smooth variant of Hughes's model for pedestrian crowd dynamics. We regularized the original model, composed by an eikonal 
equation and a continuity equation. First by a local interaction term, which intermediates individual pointwise path optimization towards conviction-weighted walking directions. Secondly, we allowed pedestrians to stop, if they are undecided, using a smooth approximation of the normalization condition. Most importantly, we restrict the information on the global density each pedestrian can use for her planning algorithm to a local surrounding area. This is a very realistic assumption for large crowds that has not been considered in the literature so far. We presented both a microscopic and a macroscopic version, and illustrated the model components in the one-dimensional case. In terms of analytical results, a rigorous theory for these kind of equations in multiple dimensions is currently out of reach to the best of our knowledge. However, we were able to identify some qualitative properties of the dependence of the optimal path on the vision cone that allow for a reduction of complexity.

The numerical approximation of the model on both levels has been discussed and utilizes several techniques including sweeping and marching methods, particle approximations and finite volume schemes. Though the numerical costs of computing a solution have increased due to the inhomogeneity of vision cones, we observe new effects and phenomena in the model based on our simulations. First, local groups of pedestrians are able to change repeatedly their walking direction towards an exit. This "multiple turnaround behavior" can be explained by the multiple sonic points of the estimated walking costs, which by construction cannot occur in the classical case. We stress that the smoothening and conviction terms are crucial to allow a swift turning behavior, which is not trivial to model in first-order equations. Second, the model replicates a waiting behavior in case of undecided pedestrians, i.e. in areas where locally estimated walking costs towards different exits are equal. Surprisingly, we found that this waiting phenomena induced by localized information can improve the overall evacuation performance of the crowd. In our numerical example we observed two local minima when varying the vision cone diameter.

To conclude, we have demonstrated that local vision effects can be implemented into first-order models for crowd dynamics. This leads to new unforeseen phenomena and complex behavior, whose partial understanding via qualitative properties is important for the applicability of such equations to social-economic problems. On the other hand, this work illustrates the limitations to first-order models such as Hughes', where planning decisions are instantaneously updated and no social or cognitive memory is taken into account. From our point of view Hughes'-type equations constitute an important building block for crowd models and a mathematically important object of study, but it cannot be expected to be fully realistic.

\section{Appendix A. Simulation Parameters}

\section{A.1. 1D macro parameters in Sec. 5.1}

The macroscopic simulation in $1 \mathrm{D}$ was implemented in MATLAB. The domain $[0,1]$ was uniformly discretized with $\Delta x=10^{-4}$. The time step was set to $\Delta t=5 \cdot 10^{-5}$. 
The vision cone was defined as $V_{x}=[x-L / 2, x+L / 2] \cap[0,1]$ with $L=0.75$. The radial interaction kernel $\mathcal{K}$ was chosen as the indicator function on the interval $[0,0.05]$. The smoothed projection operator was chosen as in (3.6) with $\ell=0.05$ and $k=25$. The wall repulsion $W(x)$ was neglected in 1D. Absorbing boundary conditions were applied at both exits. The cost function was numerically bounded at $c(\rho) \leq 10^{4}$.

\section{A.2. 2D micro parameters in Sec. 5.2}

The microscopic simulations are implemented using the software package Netgen/NgSolve. The domain was discretized in 1438 triangles, the time steps were set to $\Delta t=10^{-2}$, the final time to $T=1.5$. At time $t=0$ we distributed the 500 particles according to the initial datum $\rho_{0}$ used in Sec. 5.1. The empirical density $\rho_{g}^{N}$ was calculated using Gaussians with variance $\sigma=0.05$ for the smooth approximation $g$. The width of the local vision cone was set to $L=0.25$ in Figs. $6(\mathrm{a})-6(\mathrm{e})$ and to $L=0.75,0.5$ and $L=0.25$ in Fig. 6(f).

\section{A.3. 2D macro parameters in Sec. 5.3}

The macroscopic simulation in $2 \mathrm{D}$ was implemented in MATLAB. The domain $\Omega=$ $[0,1] \times\left[0, \frac{1}{2}\right]$ was uniformly discretized with $\Delta x=\Delta y=10^{-3}$. The time step was set to $\Delta t=5 \cdot 10^{-3}$. A Fast Sweeping Method was used to solve the eikonal equations. The vision cone was defined as $V_{x}=\left\{y:\|y-x\|_{2} \leq \frac{L}{2}\right\} \cap \Omega$ with varying diameter $L$. The radial interaction kernel $\mathcal{K}$ was chosen as

$$
\mathcal{K}(x)= \begin{cases}\exp \left(-\frac{b^{2}}{b^{2}-\|x\|^{2}}\right), & \|x\| \leq b, \\ 0, & \text { else, }\end{cases}
$$

with $b=0.05$. The smoothed projection operator was chosen as in (3.6) with $\ell=0.05$ and $k=25$. The wall repulsion $W(x)$ was defined with the width of the boundary layer function $\chi_{w}$ set to $w=0.025$. The wall costs $W(x)$ were numerically bounded at $W(x) \leq c(0.975)$. The cost function was numerically bounded at $c(\rho) \leq$ $10^{3}$. The numerical accuracy for vanishing density was set to $10^{-7}$.

\section{Acknowledgments}

J.A.C. acknowledges support from projects MTM2011-27739-C04-02 and the Royal Society through a Wolfson Research Merit Award. J.A.C. and S.M. were supported by Engineering and Physical Sciences Research Council (UK) Grant Number EP/K008404/1. M.T.W. acknowledges financial support from the Austrian Academy of Sciences ÖAW via the New Frontiers Group NSP-001.

\section{References}

1. D. Amadori and M. Di Francesco, The one-dimensional Hughes' model for pedestrian flow: Riemann-type solutions, Acta Math. Sci. 32 (2012) 259-280. 
2. D. Amadori, P. Goatin and M. D. Rosini, Existence results for Hughes' model for pedestrian flows, J. Math. Anal. Appl. 420 (2014) 387-406.

3. P. Amorim, R. M. Colombo and A. Teixeira, On the numerical integration of scalar nonlocal conservation laws, ESAIM: Math. Model. Numer. Anal. 49 (2015) 19-37.

4. C. Appert-Rolland, P. Degond and S. Motsch. Two-way multi-lane traffic model for pedestrians in corridors, Netw. Heterog. Media 6 (2011) 351-381.

5. M. Bardi and I. Capuzzo-Dolcetta, Optimal Control and Viscosity Solutions of Hamilton-Jacobi-Bellman Equations, Systems and Control: Foundations and Applications (Birkhäuser, 1997).

6. N. Bellomo, A. Bellouquid and D. Knopoff, From the microscale to collective crowd dynamics, Multiscale Model. Simulat. 11 (2013) 943-963.

7. N. Bellomo and C. Dogbe. On the modeling of traffic and crowds: A survey of models, speculations and perspectives, SIAM Rev. 53 (2011) 409-463.

8. N. Bellomo, B. Piccoli and A. Tosin, Modeling crowd dynamics from a complex system viewpoint, Math. Models Methods Appl. Sci. 22 (2012) 1230004, 29 pp.

9. S. Blandin and P. Goatin, Well-posedness of a conservation law with non-local flux arising in traffic flow modeling, preprint (2014), http://haal.invia.fr/hal-00954527.

10. M. Burger, M. Di Francesco, P. Markowich and M.-T. Wolfram, Mean field games with nonlinear mobilities in pedestrian dynamics, Discrete Contin. Dynam. Syst. Ser. B 19 (2014) 1311-1333.

11. C. Burstedde, K. Klauck, A. Schadschneider and J. Zittartz, Simulation of pedestrian dynamics using a two-dimensional cellular automaton, Physica A 295 (2001) $507-525$.

12. R. M. Colombo, M. Garavello and M. Lécureux-Mercier, A class of nonlocal models for pedestrian traffic, Math. Models Methods Appl. Sci. 22 (2012) 1150023.

13. R. M. Colombo and M. Lécureux-Mercier, Nonlocal crowd dynamics models for several populations, Acta Math. Sci. 32 (2012) 177-196.

14. G. Crippa and M. Lécureux-Mercier, Existence and uniqueness of measure solutions for a system of continuity equations with non-local flow, NoDEA Nonlinear Differential Equations Appl. 20 (2013) 523-537.

15. E. Cristiani, B. Piccoli and A. Tosin, Multiscale modeling of granular flows with application to crowd dynamics, Multiscale Model. Simulat. 9 (2011) 155-182.

16. P. Degond, C. Appert-Rolland, M. Moussad, J. Pettré and G. Theraulaz, A hierarchy of heuristic-based models of crowd dynamics, J. Statist. Phys. 152 (2013) 1033-1068.

17. P. Degond, C. Appert-Rolland, J. Pettré and G. Theraulaz, Vision-based macroscopic pedestrian models, Kinet. Relat. Models 6 (2013) 809-839.

18. M. Di Francesco, P. A. Markowich, J.-F. Pietschmann and M.-T. Wolfram, On the Hughes' model for pedestrian flow: The one-dimensional case, J. Differential Equations 250 (2011) 1334-1362.

19. C. Dogbé, Modeling crowd dynamics by the mean-field limit approach, Math. Comput. Model. 52 (2010) 1506-1520.

20. M. Dumbser, A. Hidalgo, M. Castro, C. Parés and E. F. Toro, FORCE schemes on unstructured meshes II: Non-conservative hyperbolic systems, Comput. Methods Appl. Mech. Engrg. 199 (2010) 625-647.

21. N. El-Khatib, P. Goatin and M. D. Rosini, On entropy weak solutions of Hughes model for pedestrian motion, Z. Angew. Math. Phys. 64 (2013) 223-251.

22. J. H. M. Evers, R. C. Fetecau and L. Ryzhik, Anisotropic interactions in a first-order aggregation model, Nonlinearity 28 (2015) 2847.

23. P. Goatin and M. Mimault, The wave-front tracking algorithm for Hughes' model of pedestrian motion, SIAM J. Sci. Comput. 35 (2013) B606-B622. 
24. D. Helbing, I. Farkas and T. Vicsek, Simulating dynamical features of escape panic, Nature 407 (2000) 487-490.

25. D. Helbing and P. Molnar, Social force model for pedestrian dynamics, Phys. Rev. E 51 (1995) 4282.

26. D. D. Holm, Geometric Mechanics. Part I, 2nd edn. (Imperial College Press, 2011).

27. S. P. Hoogendoorn and P. Bovy, Pedestrian route-choice and activity scheduling theory and models, Transport. Res. B-Meth. 38 (2004) 169-190.

28. L. Huang, S. Wong, M. Zhang, C.-W. Shu and W. H. Lam, Revisiting Hughes dynamic continuum model for pedestrian flow and the development of an efficient solution algorithm, Transport. Res. B-Meth. 43 (2009) 127-141.

29. R. L. Hughes, A continuum theory for the flow of pedestrians, Transport. Res. B-Meth. 36 (2002) 507-535.

30. R. Kimmel and J. A. Sethian, Computing geodesic paths on manifolds, Proc. Natl. Acad. Sci. USA 95 (1998) 8431-8435.

31. A. Lachapelle and M.-T. Wolfram, On a mean field game approach modeling congestion and aversion in pedestrian crowds, Transport. Res. B-Meth. 45 (2011) 1572-1589.

32. M. J. Lighthill and G. B. Whitham, On kinematic waves. II. A theory of traffic flow on long crowded roads, Proc. Ser. Roy. Soc. Lond. A Math. Phys. Engrg. Sci. 229 (1955) 317-345.

33. M. Moussaid, E. G. Guillot, M. Moreau, J. Fehrenbach, O. Chabiron, S. Lemercier, J. Pettré, C. Appert-Rolland, P. Degond and G. Theraulaz, Traffic instabilities in self-organized pedestrian crowds, PLoS Comput. Biol. 8 (2012).

34. J. Qian, Y. Zhang and H. Zhao, Fast sweeping methods for eikonal equations on triangular meshes, SIAM J. Numer. Anal. 45 (2007) 83-107.

35. P. I. Richards, Shock waves on the highway, Oper. Res. 4 (1956) 42-51.

36. J. A. Sethian, Fast marching methods, SIAM Rev. 41 (1999) 199-235.

37. E. F. Toro, A. Hidalgo and M. Dumbser, Force schemes on unstructured meshes I: Conservative hyperbolic systems, J. Comput. Phys. 228 (2009) 3368-3389.

38. A. Treuille, S. Cooper and Z. Popović, Continuum crowds, ACM Trans. Graph. 25 (2006) 1160-1168.

39. F. Venuti and L. Bruno, Crowd-structure interaction in lively footbridges under synchronous lateral excitation: A literature review, Phys. Life Rev. 6 (2009) 176-206.

40. H. Zhao, A fast sweeping method for eikonal equations, Math. Comput. 74 (2005) 603-627. 